\title{
Performance Analysis of Decode-Amplify-and- Forward Scheme used in Relay-based Cooperative Spectrum Sensing in Cognitive Radio Network
}

\author{
Hirdesh Chack \\ Department of Electronics and \\ Communication Engineering, \\ Maulana Azad National \\ Institute of Technology, \\ Bhopal, India
}

\author{
Hemendra Dhurvey \\ Department of Electronics and \\ Communication Engineering, \\ Maulana Azad National \\ Institute of Technology, \\ Bhopal, India
}

\author{
O. P. Meena \\ Department of Electronics and \\ Communication Engineering, \\ Maulana Azad National \\ Institute of Technology, \\ Bhopal, India
}

\begin{abstract}
In this paper we have analyzed performance of relay based Cognitive Radio (CR) networks. Later on we present a scheme for cooperative spectrum sensing known as DetectAmplify-and-Forward (DAF). This technique is having the capacity of sensing over non-identical Nakagami- $m$ fading channels. We have also introduced an advanced statistical approach to derive a new relation in order to calculate the average false alarm probability and average detection probability.
\end{abstract}

In subsequent section it is also proved that a small number of reliable cognitive radios are enough to achieve practical detection level in cooperative spectrum sensing instead of incorporating all CRs. The simulation results shows improvement in detection accuracy and reduction in bandwidth requirement of the relaying links by abstaining from the heavily faded relays in the DAF scheme

\section{Keywords}

Cooperative Spectrum Sensing, Cognitive radio, Energy detection. , Decode-Amplify-and-Forward.

\section{INTRODUCTION}

Spectrum sensing is a major task in cognitive networks. The spectrum sensing efficiency can be improved by cooperative spectrum sensing because fusion center collects the sensing information from many $\mathrm{CRs}$ and afterward takes final decision about the presence or absence of PU [1]. When cooperative relay based networks are used; two paths become really important first the sensing paths from the source (primary user) to the relays (CR users), and second the relaying paths from the relays to the receiver [2]. There are two types of protocols generally used in these networks either Amplify Forward (AF) protocol or Decode Forward (DF) protocol. In AF protocol measurements are sent to fusion center and In DF protocol decisions are sent to fusion center. The advantage of DF protocol is that it needs less bandwidth on the other hand AF protocol helps in reducing complexity at the local radios.

Generally two types of gain based relays are used. The relay based on amplifying gain is known as channel state information (CSI)-assisted relay and fixed-gain relay. In (CSI)-assisted relay vary gain according to the sensed power [3]. On the other hand, the fixed-gain relay amplifies and forwards the received signal with a constant gain which results in an output signal with variable power. The fixed-gain relay also reduces the complexity of relay, but CSI assisted relay can perform better when fading channels are used.
It is found by the researchers that Relay-based CR improves the performance of both spectrum sensing and secondary transmissions. It is also found that secondary users with higher detection probabilities constantly act as relays to help those with lower detection probabilities in a distributed network with i.i.d. Rayleigh fading channels is considered .

We analyse the performance DAF relaying scheme that reduces the required bandwidth. We will see that multipath fading on relaying channels yields similar performance degradations as multipath fading on sensing channels.

We will mainly analyse,

1) A Detect-Amplify-and-Forward (DAF) relaying scheme for CSS over non-identical Nakagami-m fading channels.

2) An error analysis scheme to investigate the truncating infinite series that appear in the closedform expressions of false alarm probability and detection probability.

\section{RELAY SCHEMES}

There are three main relay schemes namely as AF, DF and DAF. There are four finite sets $\mathbf{X}_{\mathbf{1}}, \mathbf{X}_{\mathbf{2}}, \mathbf{Y}_{\mathbf{1}}$, and $\mathbf{Y}$ in a single relay channel. Conditional probability distribution $p\left(y, y_{1} \mid x_{1}, x_{2}\right)$ on these sets, and the probability distribution of the choice of symbols selected by the encoder and the relay encoder is represented by $\left(x_{1}, x_{2}\right)$

\subsection{Amplify-and-Forward (AF) relay scheme}

In this relaying scheme, the relay sends an amplified received signal to the fusion center in last time slot. It requires much less delay because the relay node operates time-slot by timeslot. It also consumes less computing power because decoding or quantizing operation is performed at the relay side [4].

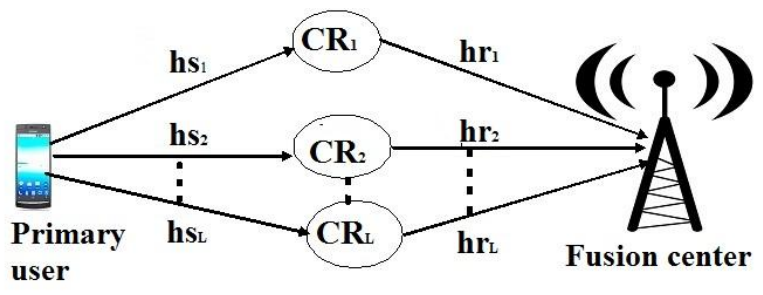

Fig.1 System model

We assume a CR network having L secondary shown in Fig.1. Where decision made by a fusion center. The 
secondary users, $\left\{C R_{i}\right\}_{i=1}$, denotes the system relays and shares the same spectrum band with the primary users. The channel fading parameter of the $i$ th sensing link is $h_{s i}$ and the $i$ th relaying link is $h_{r i}$, We also denote by and the additive Gaussian noise of the sensing channel is $n_{\boldsymbol{i}}$ and $n_{r_{\boldsymbol{i}}}$ is additive Gaussian noise of relaying channel, It is assumed to be i.i.d with zero mean and variance $N_{0}[5]$.

We It is also assumed that channel state information (CSI) is available for all the relays and fusion center has a full knowledge of CSI. it can collect knowledge of sensing and relaying channel gains. The time slot structure used is shown in Fig.2.

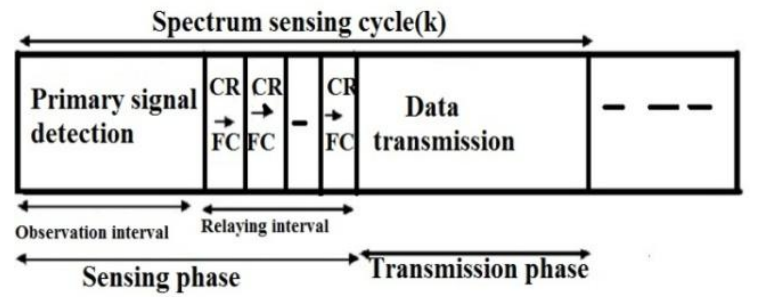

Fig.2 Time slot structure

Let $x_{p}$ denotes the signal transmitted by the primary radio, then the received signal by the $i^{\text {th }} \mathrm{CR}$ user, then $y_{S \boldsymbol{i}}$, can be expressed as

$y_{s_{i}}=\theta h_{s_{i}} x_{p}+n_{s_{i}}$

Where $\theta=0$ or 1 denotes the primary user state under two hypotheses $: H_{O}$ for primary user absence and $H_{1}$ for primary user presence.

If $Y_{S i}$ denotes the power of $y_{s i}$, then the mean value of $Y_{S i}$ can be expressed as

$E\left[Y_{s_{i}}\right]=\left\{\begin{array}{cc}\sigma_{Y_{s_{i_{1}}}}=E_{i} & H_{0} \\ \sigma_{Y_{S_{i_{1}}}}=E_{i}+N_{0}=N_{0}\left(1+\widehat{\gamma_{s_{i}}}\right) & H_{1}\end{array}\right.$

where, $E_{i}=\mathrm{E}\left\{\left|h_{s i} x_{p}\right|^{2}\right\}$ is the mean value of the signal power as received at the RF front-end of the $i$ th $C R$ receiver and $\gamma_{s_{i}}=E_{i}=N_{0}$ is the average SNR associated with the ith sensing link[6],[7],[8]. For local spectrum sensing, $Y_{S \boldsymbol{i}}$ is compared with a given threshold $\lambda_{i}$ to infer the primary state $\theta$. Hence, the false alarm probability, $P_{f_{i}}$, and the detection probability, $P_{d_{\boldsymbol{i}}}$, can be expressed as .

$P_{f_{i}}=\int_{\delta_{i}}^{\infty}\left(\frac{m_{i}}{\sigma_{Y_{s_{i}}}}\right)^{m_{i}} \frac{y^{m_{i}-1}}{\Gamma\left(m_{i}\right)} e^{\left(-\frac{m_{i}}{\sigma_{Y_{s_{i}}}}\right) y} d y$

$=\frac{\Gamma\left(m_{i}, \frac{m_{i} \lambda_{i}}{\sigma_{Y_{s_{i}}}}\right)}{\Gamma\left(m_{i}\right)}=\frac{\Gamma\left(m_{i}, \frac{m_{i} \lambda_{i}}{N_{0}}\right)}{\Gamma\left(m_{i}\right)}$

(3)

and

$$
\begin{aligned}
& P_{d_{i}}=\int_{\delta_{i}}^{\infty}\left(\frac{m_{i}}{\sigma_{Y_{s_{i}}}}\right)^{m_{i}} \frac{y^{m_{i}-1}}{\Gamma\left(m_{i}\right)} e^{\left(-\frac{m_{i}}{\sigma_{Y_{S_{i}}}}\right) y} d y \\
& =\frac{\Gamma\left(m_{i}, \frac{m_{i} \lambda_{i}}{\sigma_{Y_{s_{i}}}}\right)}{\Gamma\left(m_{i}\right)}=\frac{\Gamma\left(m_{i}, \frac{m_{i} \lambda_{i}}{N_{0}\left(1+\widehat{\gamma_{s_{i}}}\right)}\right)}{\Gamma\left(m_{i}\right)}
\end{aligned}
$$

\subsubsection{Single relay scheme}

In the AF relaying scheme, $\mathrm{CR}$ users amplify the received signal and directly relay to the fusion center [9]. It is assumed that each $\mathrm{CR}$ relay has a maximum power constraint, $P_{\boldsymbol{i}}$. Hence, it measures the average received signal power and scales it appropriately so that the power constraint is satisfied. Accordingly, the signal received at the fusion center takes the form

$$
\begin{aligned}
& y_{i}=h_{r_{i}}\left(\sqrt{ } A_{i}\left(\theta h_{s_{i}} x_{p}+n_{s_{i}}\right)\right)+n_{r_{i}} \\
= & \theta \sqrt{ } A_{i} h_{r_{i}} h_{s_{i}} x_{p}+\sqrt{ } A_{i} h_{r_{i}} n_{s_{i}}+n_{r i}
\end{aligned}
$$

Where $A_{i}$ denotes the dimensionless amplification factor of the $i$ th $\mathrm{CR}$ relay. According to the maximum power constraint, $A_{i}$ is selected as

$$
A_{i}=\frac{P_{i}}{E_{i}+N_{0}}
$$

In order to accommodate the two hypotheses $H_{0}$ and $H_{1}$, we define $g_{i}=\left|h_{r_{i}}\right|^{2}$ as the instantaneous channel gain of the $i$ th relaying link and $g_{i} \bumpeq \mathrm{E}\left\{\left|h_{r_{i}}\right|^{2}\right\}$ as the expected value of $g_{\boldsymbol{i}}$. Since $h_{\boldsymbol{r} \boldsymbol{i}}$ follows a Nakagami-m distribution, it is easy to verify that $g_{i}$ follows a gamma distribution given by

$f_{G_{i}}(g)=\left(\frac{m_{i}}{\widehat{g_{i}}}\right)^{m_{i}} \frac{g^{m_{i}-1}}{\Gamma\left(m_{i}\right)} e^{\left(-\frac{m_{i}}{\widehat{g_{i}}}\right) g}, \quad g \geq 0$

Let $Y_{i}$ denotes the power of the the relayed signal $y_{i}$, then from (5), the mean value of $Y_{i}$ for a given $g$ can be expressed as

$$
\begin{aligned}
& E\left\{Y_{i} \mid g\right\}= \\
& \left\{\begin{array}{cc}
\sigma_{Y_{i_{0}}}=N_{0}\left(1+\widehat{\theta_{i}} \sqrt{A_{i} g}\right) & H_{0} \\
\sigma_{Y_{i_{1}}}=N_{0}\left(1+\widehat{\theta_{i}}\left(1+\widehat{\gamma_{s_{i}}}\right) \sqrt{A_{i} g}\right) & H_{1}
\end{array}\right.
\end{aligned}
$$

Therefore, the probability of the false alarm for a given $g$ can be evaluated as

$$
\begin{aligned}
& P_{f_{i}}^{A F}=\left(\frac{\beta_{i}}{A_{i}}\right) \int_{\delta_{i}}^{\infty}\left(\frac{m_{i}}{\sigma_{Y_{S_{i}}}}\right)^{m_{i}} \frac{y^{m_{i}-1}}{\Gamma\left(m_{i}\right)} e^{\left(-\frac{m_{i}}{\sigma_{Y_{s_{i}}}}\right) y} d y= \\
& \frac{1}{\Gamma\left(m_{i}\right)} \Gamma\left(m_{i}, \frac{m_{i} \lambda_{i}}{N_{0}\left(1+\gamma_{i}\right)}\right)
\end{aligned}
$$

where, the integral in (9) is evaluated with the help of [31, Eq. 3.383.5] and $\lambda$ denotes the decision threshold used by the fusion center to infer the primary state $\theta$. Now, we remove the condition on $g$ and compute an average false alarm probability $P_{f i}$ by integrating over the PDF of the channel gain given in (7) as follows

Evaluating the integral in (10) as described in Appendix A.1, $P^{A F}$ can be expressed mathematically as ${\overline{P_{f_{i}}}}^{A F}=\left(\frac{B_{i}}{A_{i}}\right)^{m_{i}} \sum_{q=0}^{m_{i}-1} \frac{1}{q !}\left(\frac{m_{i} \lambda}{N_{0}}\right)^{q} \sum_{n=0}^{\infty}(-1)^{n} b_{n} \times$ 
$U\left(m_{i} ; m_{i}+1-q-n ; \frac{B_{i}}{A_{i}}\right)$

where, $\beta_{i}=m_{i} / g_{i}, b_{n}=(1 / n !)\left(m_{i} \lambda / N_{0}\right)^{n} \overline{\text {, and } U(: ; ; ;:)}$ is the confluent hyper geometric function of the second kind defined in [29, Eq. 13.1.3]. Similarly, the average detection probability $P d_{i}$ can be obtained as

$$
\begin{gathered}
\quad{\overline{P_{f_{d}}}}^{A} F=\left(\frac{B_{i}}{\left(1+\gamma_{s_{i}}\right) A_{i}}\right)^{m_{i}} \sum_{q=0}^{m_{i}-1} \frac{1}{q !}\left(\frac{m_{i} \lambda}{N_{0}}\right)^{q} \sum_{n=0}^{\infty}(-1)^{n} b_{n} \\
\times U\left(m_{i} ; m_{i}+1-q-n ; \frac{B_{i}}{\left(1+\gamma_{s_{i}}\right) A_{i}}\right)
\end{gathered}
$$

\subsubsection{Multi relay scheme}

Many performance analysis problems require determination of statistics of the sum of the squared envelopes of the faded signals over several diversity paths [10]. which can be achieved through EGC technique. For $L$ inputs, the output of the EGC receiver can be expressed as

$$
\begin{aligned}
& y=\sum_{i=0}^{L} \sqrt{A_{i}} h_{r_{i}}\left(\theta h_{s_{i}} x_{p}+n_{s_{i}}\right)+n_{r_{i}} \\
& =\theta \sum_{i=1}^{L} \sqrt{ } A_{i} h_{r_{i}} h_{s_{i}} x_{p}+\sum_{i=1}^{L} \sqrt{ } A_{i} h_{r_{i}} n_{s_{i}}+n_{r_{i}}
\end{aligned}
$$

If $Y$ denotes the power at the output of the EGC receiver, then for given $g_{i}$ 's, the mean value of $Y$ can be expressed as

$$
\begin{aligned}
& E\left\{Y \mid g_{i}{ }^{\prime} s\right\}= \\
& \left\{\begin{array}{cc}
\sigma_{Y_{0}}=N_{0}\left(1+\sum_{i=1}^{L} A_{i} g_{i}\right) & H_{0} \\
\sigma_{Y_{1}}=N_{0}\left(1+\sum_{i=1}^{L}\left(1+\overline{\gamma_{s_{i}}}\right) A_{i} g_{i}\right) & H_{1}
\end{array}\right.
\end{aligned}
$$

Over Nakagami- $m$ channel fading, the aver-age false alarm probability, $P_{f i}^{A F}$, for any number of cognitive radio relays, $L$, in a multi-relay $\mathrm{AF}$ system is given by

$$
\begin{aligned}
\bar{P}_{f}{ }^{A F}= & {\left[\prod_{l=1}^{L}\left(-\frac{\beta_{l}}{A_{l}}\right)^{m_{l}}\right] \sum_{i=1}^{L} \sum_{v=1}^{m_{i}}(-1)^{v} b_{i v} \sum_{q=0}^{m_{i}-1} \frac{1}{q !}\left(\frac{m_{i} \lambda}{N_{0}}\right)^{q} } \\
& \sum_{n=0}^{\infty}-1^{n} b_{n} U\left(v ; v+1-q-n ;\left(\frac{\beta_{i}}{A_{i}}\right)\right) . .
\end{aligned}
$$

Where

$$
\begin{aligned}
& b_{i v}=\left[\begin{array}{c}
\sum_{i_{1}=0}^{m_{i}-v-1}\left(\begin{array}{c}
m_{i}-v-1 \\
i_{1}
\end{array}\right) B_{i}{ }^{m_{i}-v-1} \sum_{i_{2}=0}^{i_{1}-1}\left(\begin{array}{c}
i_{1}-1 \\
i_{1}
\end{array}\right) B_{i}{ }^{i_{1}-1-i_{2}} \\
\sum_{i_{3}=0}^{i_{2}-1}\left(\begin{array}{c}
i_{2}-1 \\
i_{2}
\end{array}\right) B_{i}{ }^{i_{2}-1-i_{3}}
\end{array}\right] \times \\
& \frac{\Delta_{i}}{\left(m_{i}-v\right) !} \\
& \Delta_{i}=\prod_{j=1}^{L}\left(\frac{\beta_{i}}{A_{i}}-\frac{\beta_{j}}{A_{j}}\right)^{m_{j}}
\end{aligned}
$$

and

$$
B^{t}{ }_{i}=(-1)^{t+1} t ! \sum_{j=1}^{L} m_{i}\left(\frac{\beta_{i}}{A_{i}}-\frac{\beta_{j}}{A_{j}}\right)^{-(t+1)}
$$

If the diversity paths have i.i.d Nakagami- $m$ fading, then the average false alarm probability $P f$ and the average detection probability $P_{d}$ can be expressed as

$$
{\overline{P_{d}}}^{-A F}=\left[\prod_{l=1}^{L}\left(-\frac{B_{l}}{\left(1+\overline{\gamma_{s}}\right) A_{l}}\right)^{m_{l}}\right] \sum_{i=1}^{L} \sum_{v=1}^{m_{i}}(-1)^{v}
$$

$$
\begin{array}{r}
\times b_{i v} \sum_{q=0}^{m_{i}-1} \frac{1}{q !}\left(\frac{m_{i} \lambda}{N_{0}}\right)^{q} \sum_{n=0}^{\infty}(-1)^{n} b_{n} \\
\times U\left(v ; v+1-q-n ; \frac{\beta_{i}}{\left(1+\overline{\gamma_{s l}}\right) A_{i}}\right)
\end{array}
$$

Over Rayleigh fading channels, $P_{\text {fray }}^{A F}$ and $P_{\text {dray }}^{A F}$ is evaluated [11],[12] by setting $m_{j}=1 i=1 \ldots$ L in equation (17) and (18)

$$
\begin{aligned}
& \bar{P}_{f \text { Ray }}{ }^{A F}=(-1)^{L+1} \prod_{l=1}^{L} \frac{1}{A_{l} \bar{g}_{l}} \sum_{i=1}^{L} \bar{\Delta}_{i} \sum_{n=0}^{\infty}(-1)^{n} \\
& \widetilde{\times b_{n}} U\left(1 ; 2-n ; \frac{1}{A_{i} \bar{g}_{i}}\right)
\end{aligned}
$$

$$
\begin{aligned}
& \bar{P}_{d R a y}{ }^{A F}=(-1)^{L+1} \prod_{l=1}^{L} \frac{1}{\left(1+\bar{\gamma}_{s l}\right) A_{l} \bar{g}_{l}} \sum_{i=1}^{L} \bar{\Delta}_{i} \sum_{n=0}^{\infty}(-1)^{n} \\
& \times \tilde{b}_{n} U\left(1 ; 2-n ; \frac{1}{\left(1+\overline{\gamma_{s l}}\right) A_{i} \bar{g}_{i}}\right){ }_{\text {Where }} \\
& \bar{\Delta}_{\boldsymbol{i}}=\prod_{\boldsymbol{j}=\mathbf{1}}^{L}\left(\left(\frac{\mathbf{1}}{\boldsymbol{A}_{\boldsymbol{i}} \overline{\boldsymbol{g}}_{i}}\right)-\left(\frac{\mathbf{1}}{\boldsymbol{A}_{\boldsymbol{j}} \bar{g}_{j}}\right)\right)
\end{aligned}
$$

\subsection{Decode-and-Forward (DF) relay scheme} In this scheme, the relay decodes the message in one block and transmits the re-encoded message in the next block. The achievable rate of DF can be expressed as $\max _{p\left(x_{1}, x_{2}\right)} \min \left(I\left(x_{1} ; y_{1} \mid x_{2}\right), I\left(x_{1}, x_{2} ; y\right)\right)$

\subsection{Decode-Amplify-and-Forward (DAF) relay scheme}

In this relay scheme $C R$ detects received signal in the observation interval. If the decision is that PU is abscent, the CR keeps quiet and transmits only an indicator signal to the fusion center during its relaying time slot. Otherwise, If the $\mathrm{PU}$ is present, the CR amplifies the signal and forwards it to the fusion center.

\subsubsection{Single relay scheme}

According to the DAF scheme, every CR user performs detection and takes its own decision $\boldsymbol{\theta}_{\boldsymbol{i}}$ about the primary user. Then, if $\boldsymbol{\theta}_{\boldsymbol{i}}=1$, the CR user amplifies and relays its local sensing to the fusion center. Otherwise, it keeps quiet if $\boldsymbol{\theta}_{\boldsymbol{i}}=\mathbf{0}$.

The signal received at fusion center at $i^{\text {th }}$ link [13]

$$
\begin{gathered}
y_{i}=\widehat{\theta}_{i} h_{r_{i}}\left(\sqrt{A_{i}\left(\theta h_{s_{i}} x_{p}+n_{s_{i}}\right)}\right)+n_{r_{i}} \\
=\theta \theta_{i} \sqrt{ } A_{i} h_{r_{i}} x_{p}+\widehat{\theta}_{i} \sqrt{ } A_{i} h_{r_{i}} h_{s_{i}} x_{p}+\widehat{\theta}_{i} \sqrt{ } A_{i} h_{r_{i}} n_{s_{i}}+n_{r_{i}}(19)
\end{gathered}
$$

And

$-E\left\{Y_{i} \mid g\right\}=\left\{\begin{array}{c}\sigma_{Y_{i_{0}}}=N_{0}\left(1+\widehat{\theta}_{i} \sqrt{A_{i}} g\right) H_{0} \\ \sigma_{Y_{i_{1}}}=N_{0}\left(1+\widehat{\theta}_{i}\left(1+\widehat{\gamma_{s_{i}}}\right) \sqrt{\left.A_{i} g\right) H_{1}}\right.\end{array}\right.$

.Average false alarm probability over a Nakagami-m channel Under hypothesis $H_{0}$ can be given by 


$$
\begin{aligned}
& {\bar{P}_{f_{i}}}^{D A F}=\sum_{\hat{\theta}_{i}=0}^{\widehat{\theta}_{i}=1} P_{\left(i \mid H_{0}\right)}\left(\frac{B_{i}}{A_{i}}\right)^{m_{i}} \sum_{q=0}^{m_{i}-1}\left(\frac{m_{i} \lambda_{i}}{N_{0}}\right)^{q} \frac{1}{q !} \times \\
& \sum_{n=0}^{\infty}(-1)^{n} b_{n} U\left(m_{i} ; m_{i}+1-q-n ; \frac{B_{i}}{A_{i}}\right)
\end{aligned}
$$

Similarly, the average detection probability $P_{d i}^{D A F}$ can be expressed as

$$
\begin{aligned}
& {\overline{P_{d_{i}}}}^{D A F}=\sum_{\widehat{\theta}_{i}=0}^{\widehat{\theta}_{i}=1} P_{\left(i \mid H_{0}\right)}\left(\frac{B_{i}}{\left(1+\bar{\lambda}_{s_{i}}\right) A_{i}}\right)^{m_{i}} \sum_{q=0}^{m_{i}-1} \times\left(\frac{m_{i} \lambda_{i}}{N_{0}}\right)^{q} \frac{1}{q !} \times \\
& \sum_{n=0}^{\infty}(-1)^{n} b_{n} U\left(m_{i} ; m_{i}+1-q-n ; \frac{B_{i}}{\left(1+\bar{\lambda}_{s_{i}}\right) A_{i}}\right)
\end{aligned}
$$

where,

$$
P_{\left(i \mid H_{0}\right)}=P_{f_{i}} \widehat{\theta}_{i}\left(1-P_{f_{i}}\right)^{1-\widehat{\theta}_{i}}
$$

\subsubsection{Multi relay scheme}

In this scheme CR makes its own decision then amplifies and forwards to the fusion center only when primary user is present. The signal can be expressed as

$$
\begin{gathered}
y=\sum_{i=0}^{L} \bar{\theta}_{i} \sqrt{A_{i} h_{r i}\left(\theta h_{s i} x_{p}+n_{s i}\right)}+n_{r_{i}} \\
=\sum_{i=0}^{L} \bar{\theta}_{i} \sqrt{ } A_{i} h_{r i} h_{s i} x_{p}+\sum_{i=0}^{L} \bar{\theta}_{i} \sqrt{ } A_{i} h_{r i} n_{s i}+n_{r_{i}}
\end{gathered}
$$

for given $g_{i}$ 's[14], the mean value of $Y$ can be expressed

$$
\begin{aligned}
& E\left\{Y_{i} \mid g\right\}= \\
& \left\{\begin{array}{c}
\sigma_{Y_{i_{0}}}=N_{0}\left(1+\widehat{\sum_{i=1}^{L} \theta_{i}} \sqrt{A_{i} g}\right) H_{0} \\
\sigma_{Y_{i_{1}}}=N_{0}\left(1+\widehat{\theta}_{i}\left(1+\sum_{i=1}^{L} 1+\widehat{\gamma_{s_{i}}}\right) \theta_{i} A_{i} g\right) H_{1}
\end{array}\right\}
\end{aligned}
$$

Average false alarm probability over a Nakagami-m channel Under hypothesis $H_{0}$ can be given by

$$
\begin{gathered}
\bar{P}_{f}^{D A F}=\sum_{j=1}^{2^{L}}\left[\prod_{i=1}^{L} P^{j}{ }_{\left(i \mid H_{0}\right)}\right]\left[\prod_{l=1, \widehat{\theta}_{l}^{j} \neq 0}^{L}\left(-\frac{\beta_{l}}{A_{l}}\right)^{m_{l}}\right] \times \\
\sum_{i=1, \widehat{\theta}_{i} \neq 0} \sum_{v=1}^{m_{i}}(-1)^{v} b_{i v} \sum_{q=0}^{m_{i}-1} \frac{1}{q !}\left(\frac{m_{i} \lambda}{N_{0}}\right)^{q} \\
\sum_{n=0}^{\infty}(-1)^{n} b_{n} U\left(v ; v+1-q-n ; \frac{B_{i}}{A_{i}}\right)
\end{gathered}
$$

Similarly, the average detection probability $P_{d i}^{D A F}$ can be expressed as [15]

$$
\begin{gathered}
\bar{P}_{d i}^{D A F}=\sum_{j=1}^{2^{L}}\left[\prod_{i=1}^{L} P^{j}{ }_{\left(i \mid H_{0}\right)}\right]\left[\prod_{l=1, \widehat{\theta}_{l} \neq \neq 0}^{L}\left(-\frac{\beta_{l}}{\left(1+\overline{\gamma_{s l}}\right) A_{l}}\right)^{m_{l}}\right] \\
\times \sum_{i=1, \widehat{\theta}_{i} \neq 0}^{L} \sum_{v=1}^{m_{i}}(-1)^{v} b_{i v} \sum_{q=0}^{m_{i}-1} \frac{1}{q !}\left(\frac{m_{i} \lambda}{N_{0}}\right)^{q} \\
\sum_{n=0}^{\infty}(-1)^{n} b_{n} U\left(v ; v+1-q-n ; \frac{B_{i}}{\left(1+\overline{\gamma_{s l}}\right) A_{i}}\right)
\end{gathered}
$$

For Rayleigh fading channels $P_{\boldsymbol{f}_{\text {Ray }}}$ and $P_{d \boldsymbol{R} \text { ay }}$ are evaluated by setting $m_{i}=1 ; i=1 ; \cdots ; L$ in (25) and (26)

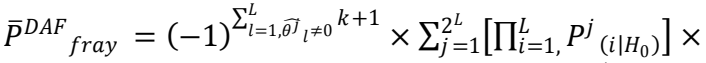

$$
\begin{aligned}
& \prod_{i=1, \widehat{\theta}_{i} \neq 0}^{L} \frac{1}{A_{l} \bar{g}_{l}} \sum_{i=1, \widehat{\theta}_{i} \neq 0}^{L} \tilde{\Delta}_{l} \sum_{n=0}^{\infty}(-1)^{n+1} \tilde{b}_{n} U(1 ; 2- \\
& \left.n ; \frac{1}{A_{i} \bar{g}_{i}}\right) \\
& \begin{array}{r}
\overline{\boldsymbol{P}}_{\text {fray }}^{\text {DAF }}=(-\mathbf{1})^{\sum_{l=1, \widehat{\theta}_{l} \neq \mathbf{0}} k+\mathbf{1}} \times \sum_{j=\mathbf{1}}^{2^{L}}\left[\prod_{i=1,}^{L} \boldsymbol{P}^{j}{ }_{\left(i \mid H_{0}\right)}\right] \\
\times \prod_{i=1, \widehat{\theta}_{i} \neq 0}^{L} \frac{1}{\left(1+\overline{\gamma_{s l}}\right) A_{l} \bar{g}_{l}}
\end{array}
\end{aligned}
$$

$$
\times \sum_{i=1, \bar{\theta}_{i} \neq 0}^{L} \tilde{\Delta}_{l} \sum_{n=0}^{\infty}(-1)^{n+1} \tilde{b}_{n} U\left(1 ; 2-n ; \frac{1}{\left(1+\overline{\gamma_{s l}}\right) A_{i} \bar{g}_{i}}\right)(28)
$$

\section{APPROXIMATION OF AVERAGE PROBABILITIES}

\subsection{Approximated probability of false alarm and detection for $\mathrm{AF}$ scheme}

Approximated probability of false alarm and probability of detection for AF scheme can be given by [16].

$$
\begin{aligned}
& \bar{P}_{f}^{A F_{f}}= \\
& 2\left[\prod_{l=1}^{L}\left(-\frac{\beta_{l}}{A_{l}}\right)^{m_{l}}\right] \sum_{i=1}^{L} \sum_{v=1}^{m_{i}} \frac{(-1)^{v} b_{i v}}{\Gamma(\mathrm{v})} \times \\
& \sum_{q=0}^{m_{i}-1} \frac{1}{q !}\left(\frac{m_{i} \lambda}{N_{0}}\right)^{\frac{v+q}{2}}\left(\frac{A_{i}}{\beta_{i}}\right)^{\frac{v-q}{2}} K_{q-v}\left(2 \sqrt{\frac{m_{i} \beta_{i} \lambda}{N_{0} A_{i}}}\right) \\
& \bar{P}^{A F}{ }_{d}=2\left[\prod_{l=1}^{L}\left(-\frac{\beta_{l}}{\left(1+\overline{\gamma_{s l}}\right) A_{l}}\right)^{m_{l}}\right] \sum_{i=1}^{L} \sum_{v=1}^{m_{i}} \frac{(-1)^{v} b_{i v}}{\Gamma(\mathrm{v})} \times \\
& \sum_{q=0}^{m_{i}-1} \frac{1}{q !}\left(\frac{m_{i} \lambda}{N_{0}}\right)^{\frac{v+q}{2}}\left(\frac{\left(1+\overline{\gamma_{s l}}\right) A_{i}}{\beta_{i}}\right)^{\frac{v-q}{2}} K_{q-v}\left(2 \sqrt{\frac{m_{i} \beta_{i} \lambda}{N_{0}\left(1+\overline{\gamma_{s l}}\right) A_{i}}}\right)
\end{aligned}
$$

where, $K_{v}(:)$ is the $v$-order modified Bessel function of the second kind.

\subsection{Approximated probability of false alarm and detection for DAF scheme}

Approximated probability of false alarm and probability of detection for DAF scheme can be given by

$$
\begin{aligned}
& \bar{P}_{f}^{D A F}=\sum_{j=1}^{2^{L}}\left[\prod_{i=1}^{L} P^{j}{ }_{\left(i \mid H_{0}\right)}\right]\left[\prod_{l=1, \widehat{\theta}^{\top}}^{L} \neq 0\right. \\
& \left.l\left(-\frac{\beta_{l}}{A_{l}}\right)^{m_{l}}\right] \times \\
& \sum_{i=1, \widehat{\theta}_{i} \neq 0}^{L} \sum_{v=1}^{m_{i}} \frac{(-1)^{v} b_{i v}}{\Gamma(\mathrm{v})} \sum_{q=0}^{m_{i}-1} \frac{1}{q !}\left(\frac{m_{i} \lambda}{N_{0}}\right)^{\frac{v+q}{2}}\left(\frac{A_{i}}{\beta_{i}}\right)^{\frac{v-q}{2}} K_{q-v}\left(2 \sqrt{\frac{m_{i} \beta_{i} \lambda}{N_{0} A_{i}}}\right)
\end{aligned}
$$

$$
\begin{aligned}
& \bar{P}^{D A F}{ }_{d} \\
& =\sum_{j=1}^{2^{L}}\left[\prod_{i=1}^{L} P^{j}{ }_{\left(i \mid H_{0}\right)}\right]\left[\prod_{l=1, \widehat{\theta}^{I_{l}} \neq 0}^{L}\left(-\frac{\beta_{l}}{\left(1+\overline{\gamma_{s l}}\right) A_{l}}\right)^{m_{l}}\right] \\
& \times \sum_{i=1, \widehat{\theta}_{i} \neq 0}^{L} \sum_{v=1}^{m_{i}} \frac{(-1)^{v} b_{i v}}{\Gamma(\mathrm{v})} \sum_{q=0}^{m_{i}-1} \frac{1}{q !}\left(\frac{m_{i} \lambda}{N_{0}}\right)^{\frac{v+q}{2}} \\
& \left(\frac{\left(1+\overline{\gamma_{s l}}\right) A_{i}}{\beta_{i}}\right)^{\frac{v-q}{2}} K_{q-v}\left(2 \sqrt{\frac{m_{i} \beta_{i} \lambda}{N_{0}\left(1+\bar{\gamma}_{s l}\right) A_{i}}}\right)
\end{aligned}
$$

\section{RESULTS AND PERFORMANCE}

\section{ANALYSIS}

To test the accuracy of the derived expressions and the performance of novel CR network method we perform simulations. The noise variance $\mathrm{NO}$ and $\mathrm{Ei}$ is unity $(0 \mathrm{~dB})$ and an upper bound of Pfi $\leq 0: 1$ and a lower bound of Pdi $\geq 0: 9$ 
are considered.

Fig. 3 shows probability of missed detection $(1-P d)$ versus probability of false alarm graph. Variety of diversity paths Are used to plot ROC curves of the energy detector. the values average false alarm probability and the average detection probability are computed from Equations (25) and (26) One can see from Fig. 3 that when the number of cooperative users increases The performance of the energy detector significantly improves. The case with $L$ $=1$ corresponds to the no-diversity scenario (single-relay system).

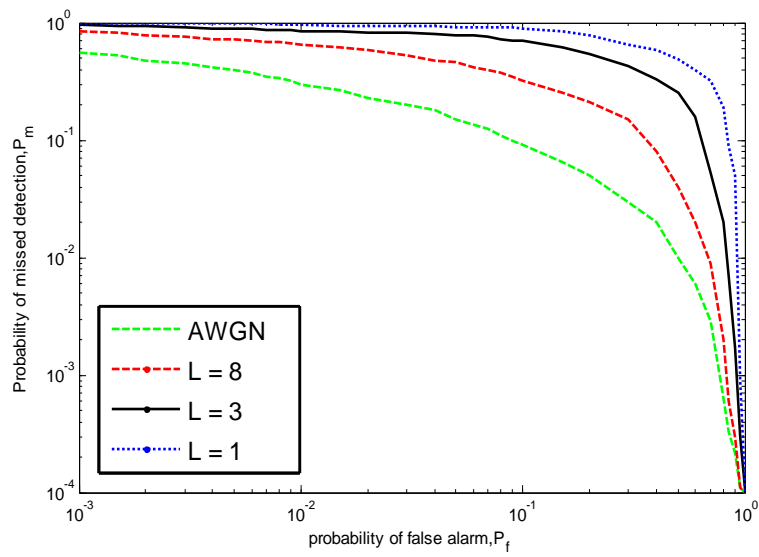

Figure 3: ROC curves of the energy detector with $m=2, P$ $=5 \mathrm{~dB}$, and $s=0 \mathrm{~dB}$.

In Fig.4 we plot $P_{d}^{A F}$ and $P_{d}^{D A F}$ for different number of cooperative users to compare the performance of the diversity system between AF and DAF schemes, we can that the detection accuracy is greatly improved when the number of cooperative users increases for both schemes. But, the DAF scheme performs better than the $\mathrm{AF}$ scheme for all the values.

This is because the cooperative users who have reliable detection probability are allowed to forward their local measurements to the fusion center. The difference in the performance of the $\mathrm{DAF}$ and $\mathrm{AF}$ strategies becomes more visible as the number of cooperative users increases. But if the number of the heavily faded users becomes large enough to induce a missed detection in the final decision the performance of system suffers .

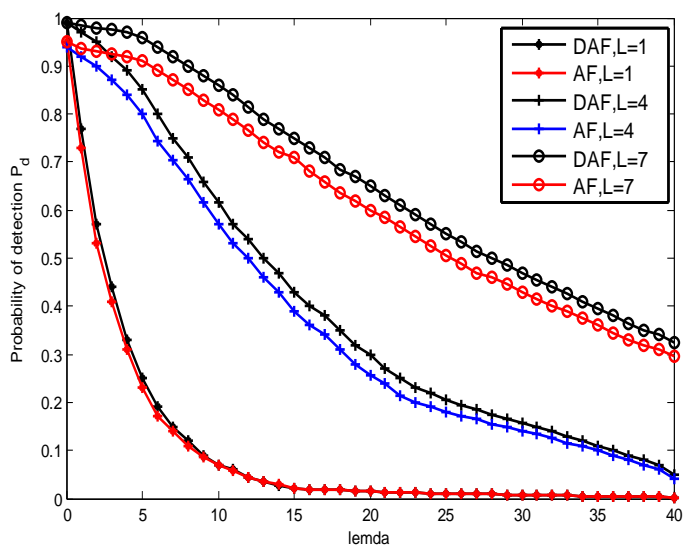

Figure 4: Detection performance of $\mathrm{AF}$ and DAF strategies for different diversity scenarios with $m=2, P=$ $5 \mathrm{~dB}$, and $s=0 \mathrm{~dB}$.
In Fig.5, the average detection probability is plotted versus Nakagami- $m$ fading scenarios with the case $m=1$ corresponds to Rayleigh fading. Three scenarios are used. For higher values of $m$ performance improves the fading severity is inversely proportional with the fading parameter, $m$. One can also see that the DAF scheme shows a better performance compared to the AF scheme, specifically at sever fading environments.

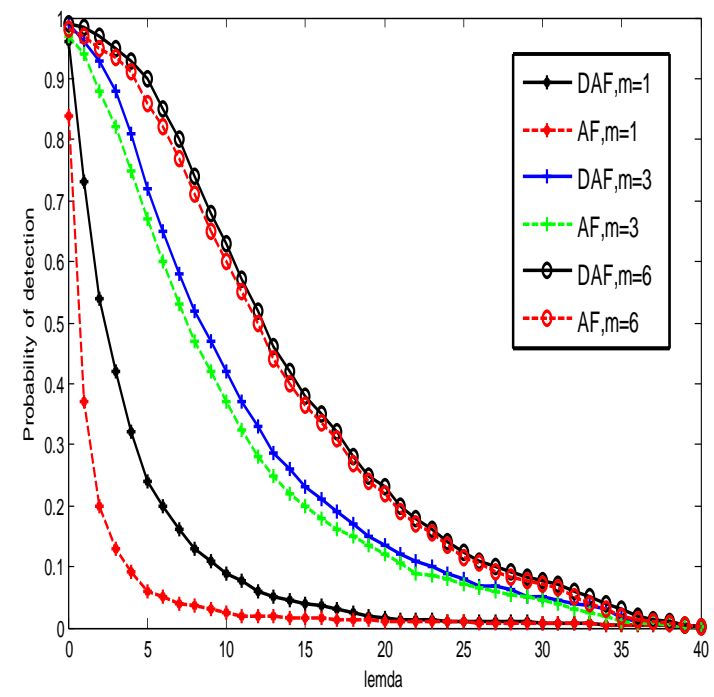

Figure 5: Detection performance of $\mathrm{AF}$ and $\mathrm{DAF}$ strategies for different fading scenarios with $L=3, P=5$ $\mathrm{dB}$, and $s=0 \mathrm{~dB}$.

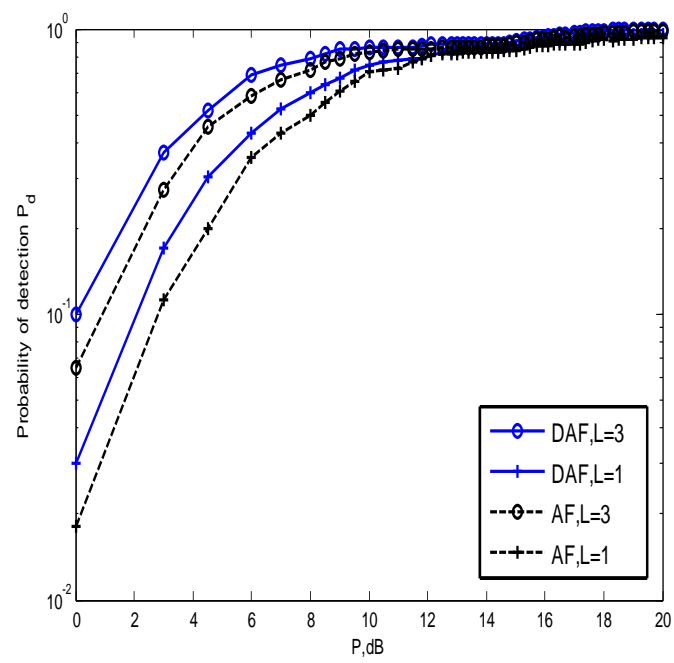

Figure 5: Detection performance of $\mathrm{AF}$ and $\mathrm{DAF}$ strategies for different power constraints with $P=5 \mathrm{~dB}$, $\mathrm{m}=2$, and $s=0 \mathrm{~dB}$.

Fig. 6 shows that the detection accuracy is can be improved by increasing the relay power constraint, $P$. The DAF scheme performs better than AF scheme for all values of the power constraint. This is because the heavily faded relays improve the detection accuracy. At higher values of $P$ the AF performance becomes very close to the DAF performance 


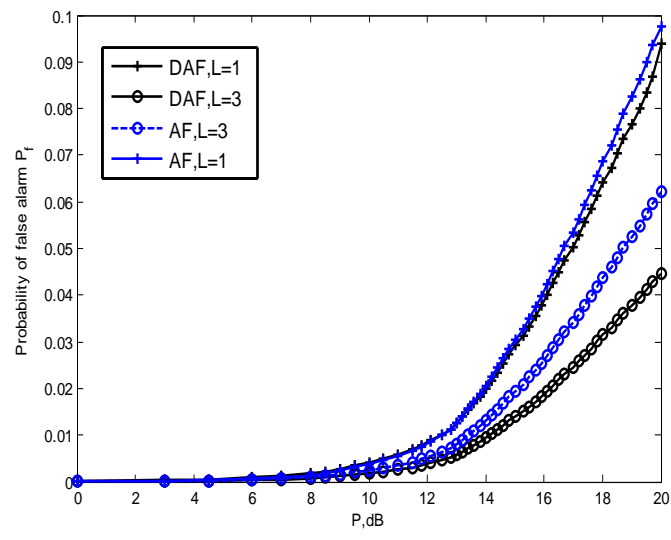

Figure 7: Probability of false alarm performance of AF and DAF strategies for different power constraints with $P$ $=5, \mathrm{~m}=2$, and $s=0 \mathrm{~dB}$.

Fig. 7 shows that an upper bound of $P f<0.1$ can be achieved for $\lambda=5$ over the selected range of the power constraint. For the cooperative case $(L>1)$, the DAF strategy performs better than the AF strategy because it eliminates the negative contributions of the unreliable users.

\section{CONCLUSIONS}

The above results and analysis shows the importance of including the relaying links and the combining techniques into the performance analysis of CR networks. The novel approach to improve detection accuracy. The derived expressions helps in improving detection accuracy over non identical fading channel because it can be used to determine the energy threshold value, the minimum number of collected energy samples, and the maximum transmission power . The shows that multipath fading heavily contributes to the unreliability of primary user detection

The results also shows that independently faded radios to collectively can achieve robustness to severe fades, multipath fading on relaying channels performs identical degradations as multipath fading on sensing channels, a small number of radios can achieve desired practical detection levels, performance measures strongly depend on the target probability of detection for dealing with fading.

BY analytical and simulation results, we can say that the derived closed-form expressions are accurate. Moreover, we analyzed that detection accuracy varies with the number of diversity branches, the fading severity, and the relay power constraint.

\section{FUTURE SCOPE}

The results give a basic knowledge upon which more comprehensive models of cooperative sensing can be developed for spectrum sharing in dynamic spectrum access systems.

\section{REFERENCES}

[1]. Q. Chen and M. Motani, "Cooperative spectrum sensing strategies for cognitive radio mesh networks," IEEE Trans. Sel. Topics Signal Process. vol. 5, no. 1, pp. 5667, Feb. 2011

[2]. Y. Zou, J. Zhu, and B. Zheng, "A cooperative sensing based cognitive relay transmission scheme without a dedicated sensing relay channel in cognitive radio networks", IEEE Trans. Signal Process., vol. 59, no. 2 , pp. 854-858, Feb. 2011

[3]. W. Zhang and K. B. Letaief, "Cooperative spectrum sensing with transmit and relay diversity in cognitive radio networks", IEEE Trans. Wireless Communications., vol. 7, no. 12, pp. 4761-4766, Dec. 2008.

[4]. Y. Zhao, R. Adve, and T. J. Lim, "Improving amplifyand-forward relay networks: optimal power allocation versus selection", IEEE Trans. Wireless. Comm., vol. TWC-6, August 2007

[5]. Sattar Hussain and Xavier N. Fernando, "Performance Analysis of Relay-Based Cooperative Spectrum Sensing in Cognitive Radio Networks Over Non-Identical Nakagami- $m$ Channels", IEEE Transactions on communications, vol. 62 , no. 8 , august 2014

[6]. M. Nakagami, "The m-distribution: A general formula of intensity distributionof rapid fading", StatisticalMethods in RadioWave Propagation. New York, NY, USA: Pergamon, 1960

[7]. I. Trigui, A. Laourine, S. Affes, and A. Stéphenne, "Performance analysis of mobile radio systems over composite fading/shadowing channels with co-located interference", IEEE Trans. Wireless Commun., vol. 8, no. 7, pp. 3448-3453, Jul. 2009.

[8]. M. Abdel-Hafez and M. Safak, "Performance analysis of digital cellular systems in Nakagami fading and correlated shadowing environmental", IEEE Trans. Veh. Technol., vol. 48, no. 5, pp. 1381-1391,Sep. 1999.

[9]. S. Borade, L. Zheng, and R. Gallager, "Amplify-andforward in wireless relay networks: Rate, diversity, and network size", IEEE Trans. Inform. Theory, vol. IT-53, October 2007

[10].Q. You, Z. Chen, Y. Li, and B. Vucetic, "Multi-hop bidirectional relay transmission schemes using amplifyand-forward and analog network coding", To appear in Proc. IEEE ICC 2011, Kyoto, Japan, June 2011.

[11].M. K. Simon and M.-S. "Alouini, Digital Communication Over Fading Channels", 2nd ed. New York, NY, USA: Wiley, 2005.

[12].O.P. Meena and Ajay somkuwar "Analysis of Frequency Division Spectrum Sensing in Cognitive Radio Network for Nakagami Fading Channel", International Journal of Computer Applications (0975 - 8887) Volume 114 - No. 3, March 2015.

[13]. M. Abramowitz and I. A. Stegun, "Handbook of Mathematical Functions with Formulas, Graphs, and Mathematical Tables", 9th ed. New York, NY, USA: Dover, 1972.

[14].S. Gradshteyn and I. M. Ryzhik, "Table of Integrals, Series, and Products", 6th ed. London, U.K.: Academic, 2000.

[15].P. Wynn, "Acceleration techniques in numerical analysis, with particular reference to problems in one independent variable", in Proc. IFIPS, Munich, 1962, pp. 149-156. 